\title{
Headache, fever and back pain in a 16-year-old boy
}

\author{
Alireza Nateghian MD, Vivek Mehta MD, Joan L Robinson MD
}

\section{CASE PRESENTATION}

A previously healthy 16-year-old boy of East Indian origin was admitted to the Stollery Children's Hospital (SCH) in Edmonton, Alberta in February 2003 for evaluation of headache and back pain. Lower back pain had started suddenly nine days earlier, after lifting weights, and had persisted. A throbbing occipital headache started eight days before admission. The patient thought he had possibly been febrile.

There was no known exposure to tuberculosis and no history of ill contacts. There had been recent travel to California and visitors from India had been staying in the home. The boy had always lived in Canada.

One day before admission to the $\mathrm{SCH}$, the boy was evaluated in a regional hospital because of worsening headache and back pain. Temperature was $38^{\circ} \mathrm{C}$, pulse was 84 beats $/ \mathrm{min}$, respiratory rate was 18 breaths/min and blood pressure was 120/80 mmHg.

Physical examination revealed a coherent boy in mild distress. He vomited for the first time during the assessment. His neck was stiff and a positive Brudzinski sign was present. Fundoscopy was normal. Tympanic membranes, mouth and throat were normal. No significant lymphadenopathy was noted. Chest, cardiac and abdominal examinations were unremarkable. Cranial nerves, sensation, tone, power, coordination and deep tendon reflexes were normal. On examination of the muskuloskeletal system, there was slight tenderness over the spinus processes of the third and fourth lumbar vertebrae (L3 and L4).
A noncontrast brain computerized tomography scan was normal. Examination of cerebrospinal fluid (CSF) revealed a white blood cell count of $\left.6.1 \times 10^{9} / \mathrm{L}\right)$ with a differential as follows: $74 \%$ polymorphonuclear, 10\% lymphocytes, $16 \%$ monocyte/macrophage; red blood cells $2.50 \times 10^{12} / \mathrm{L}$; total protein $2.82 \mathrm{~g} / \mathrm{L}$; glucose $1 \mathrm{mmol} / \mathrm{L}$ (concurrent blood glucose $5 \mathrm{mmol} / \mathrm{L})$. A complete blood count revealed a white blood cell count of $10.3 \times 10^{9} / \mathrm{L}$ with $80 \%$ neutrophils, $12 \%$ lymphocytes, $7 \%$ monocytes and $1 \%$ basophils. Hemoglobin was $14.6 \mathrm{~g} / \mathrm{L}$ and platelets were $239 \times 10^{9} / \mathrm{L}$. Chemistry panel and urinalysis were normal and chest radiograph (both lateral and posteroanterior views) were normal. Parenteral cefotaxime and vancomycin were started.

The following day, the boy was transferred to the SCH for further diagnostic work-up because the CSF and blood cultures yielded no growth and the patient's symptoms persisted. On admission, physical examination was unchanged from the previous day. A tuberculin skin test was done (and was ultimately negative). The vancomycin was stopped. Over the next $48 \mathrm{~h}$, the headache improved but the back pain and fever persisted. This pain now involved only the lower back and the patient remained slightly tender in the mid-line in the region of L3 to L4. Staphylococcus aureus was isolated from the CSF culture in broth after $72 \mathrm{~h}$ but there was no growth on the primary agar plates. The parenteral cefotaxime was changed to cloxacillin. A diagnostic test was performed. What was the diagnosis?

Department of Pediatrics and Stollery Children's Hospital, University of Alberta, Edmonton, Alberta

Correspondence: Dr Joan L Robinson, 2C3 Walter Mackenzie Centre, University of Alberta, Edmonton, Alberta T6G $2 B 7$.

Telephone 780-407-1680, fax 780-407-7136, e-mail jr3@gpu.srv.ualberta.ca 


\section{DIAGNOSIS}

Magnetic resonance imaging (MRI) of the lumbar spine with and without augmentation revealed an intradural, extramedullary oval rim-enhancing collection in the posterior canal at the L4 level, which was thought to be an abscess. There was also enhancement of the cauda with no evidence of discitis, osteomyelitis or paraspinal abscess (Figure 1).

A partial L2 and complete L3 to L5 bilateral laminectomy were performed. Despite the absence of any external manifestations, there was a sinus tract which went between the L5 and S1 discs down to the dura and led to an inflamed and ruptured epidermoid cyst $(2.0 \times 0.7 \mathrm{~cm}$ in size $)$ in the intraduralextramedullary space. Pathological study of excised material demonstrated a chronically inflamed epidermoid cyst with no inflammation in the sinus tract. Gram staining of material from the cyst showed no bacteria and the cultures yielded no growth.

\section{DISCUSSION}

Spinal subdural or intradural abscess are rare (1). Most subdural abscesses occur in the brain, and paraspinal abscesses are usually epidural. Presence of the wide epidural space as a protective filter for the subdural space and centripetal fashion of blood flow in the region of the spinal cord may explain the rarity of spinal subdural abscesses (SSA) (2). The majority of published case reports of spinal abscesses beneath the dura are intramedullary $(1,3)$ in comparison with our case, which was extramedullary.

Most spinal dermoid and epidermoid cysts are developmental but they can also arise by doing a lumbar puncture (LP) without a stylet (4). They can be occult (as in our case) or can connect to the skin via a dermoid sinus. These sinuses can extend from the skin to the intramedullary region of the spinal cord and can result in abscess formation anywhere along their tract. A recent series of pediatric patients with intramedullary abscesses found that almost half were associated with dermal sinuses but it is less clear how frequently the extramedullary SSA are related to anatomic defects $(1,3)$. The diagnostic test of choice for paraspinal abscesses is MRI. Occasionally, however, negative studies will be found if the problem is a dermoid or epidermoid cyst that has recently ruptured (4).

Infection of the subdural space can occur from hematogenous spread, from direct spread of contiguous infection or from procedures such as an LP (2). The organisms most commonly isolated from SSA are S aureus, Staphylococcus epidermidis, Streptococcus milleri and, to a lesser degree, Escherichia coli and Bacteroides species $(1,2,5)$. Because $S$ aureus is such an unusual cause of meningitis, it has been suggested that one should always search for a neurocutaneous fistula in patients with $S$ aureus meningitis (4). In our case, the growth of $S$ aureus from the CSF in broth culture could be from contamination of

\section{REFERENCES}

1. Chen-Yin C, Kuang-Lin L, Huei-Shyong W, et al. Dermoid cyst with dermal sinus tract complicated with spinal subdural abscess. Ped Neurol 1999;20:157-60.

2. Schneider P, Givens T. Spinal subdural abscess in a pediatric patient: A case report and review of the literature. Ped Emerg Care 1998,14:22-4.

3. Simon J, Lazareff J, Diament M, et al. Intramedullary abscess of the spinal cord in children: A case report and review of the literature. Ped Inf Dis J 2003;22:186-92.

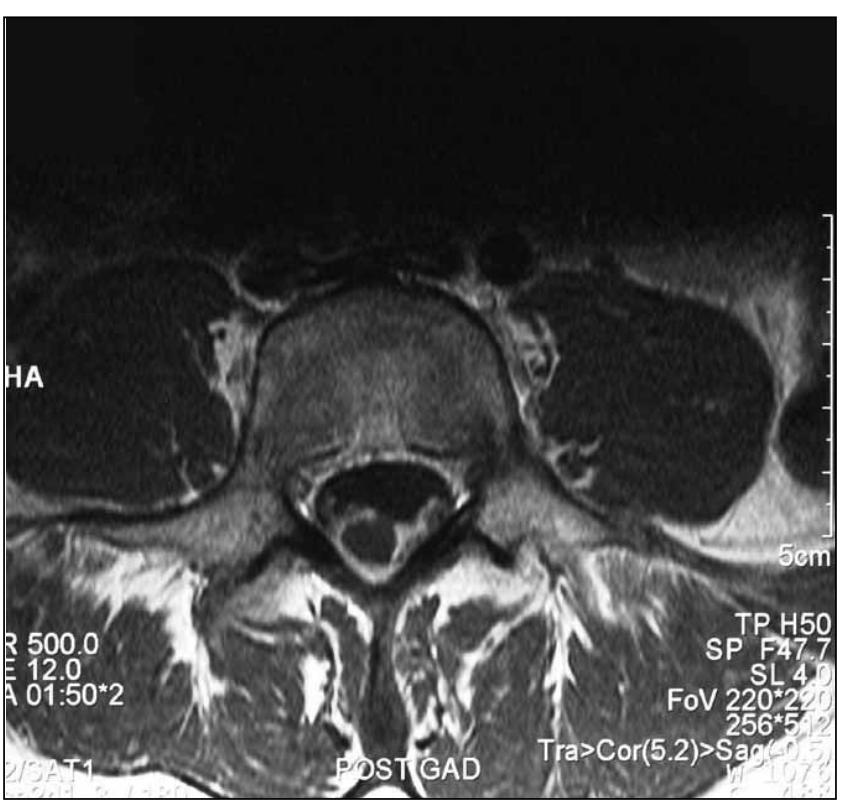

Figure 1) Magnetic resonance imaging of patient (after gadolinium infusion), showing an intradural, extramedullary oval rim-enhancing abnormality in the posterior canal at the L4 level

the needle when passed through the skin, from contamination of the needle when passed through the epidermoid cyst (although the site of the LP appeared to be higher than that of the cyst) or most likely from actual rupture of an infected cyst into the CSF.

All spinal dermoid and epidermoid cysts should be excised. Urgent surgical drainage followed by appropriate antibiotic therapy is the usual management of paraspinal abscesses $(1,6)$. Treatment of paraspinal abscesses with antibiotics alone often results in a poor outcome $(1,2)$. In our case where the SSA was small and was actually an inflamed epidermoid cyst, there may be less urgency than in other cases because of the smaller abscess size, but permanent neurological sequelae have been described in an SSA resulting from a dermoid cyst (1). With an infected spinal dermoid or epidermoid cyst and no apparent neurological signs, some investigators recommend an appropriate course of antibiotic therapy to decrease the inflammation, followed by exploration and resection of the cyst and sinus tract (5). Antibiotics are usually given for a minimum of six weeks for paraspinal abscesses, but we gave only a seven-day postoperative course of intravenous cloxacillin because the entire abscess was resected, and it was debatable if the CSF was ever infected. On follow-up one month later, physical examination was normal and the MRI showed only enhancement around the operative site.

4. Kriss TC, Kriss VM, Warf BC. Recurrent meningitis: The search for the dermoid or epidermoid tumor. Pediatr Infect Dis J 1995;14:697-700.

5. Calvit M, Aranda G. Timing of surgery in patients with infected spinal dermal sinuses: Report of two cases. Child Nerv Syst 1995;11:129-32.

6. Martin RJ, Yuan HA. Neurosurgical care of spinal epidural, subdural, and intramedullary abscesses and arachnoiditis. Orthop Clin North Am 1996;27:125-36. 


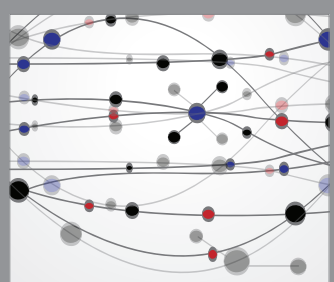

The Scientific World Journal
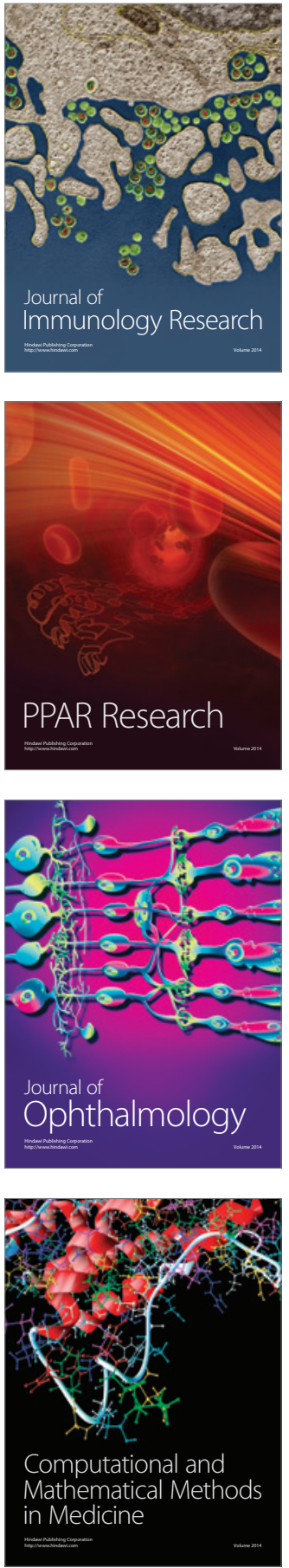

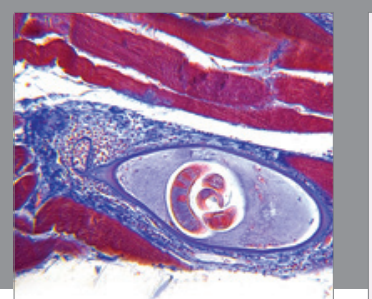

Gastroenterology Research and Practice

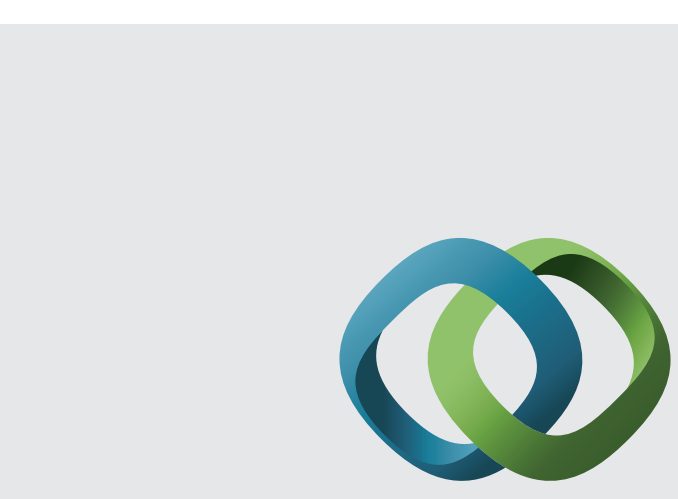

\section{Hindawi}

Submit your manuscripts at

http://www.hindawi.com
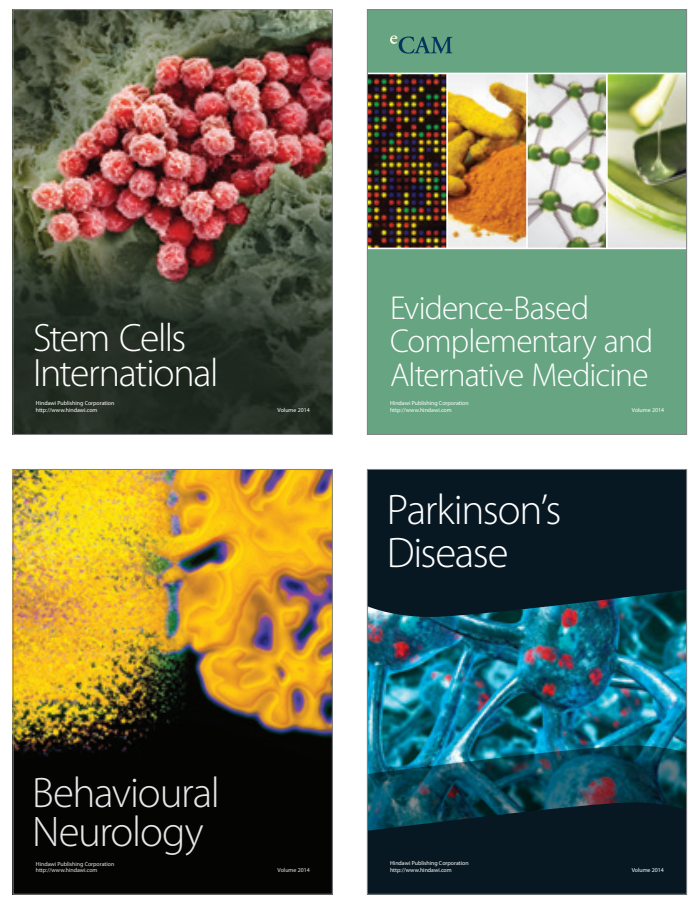
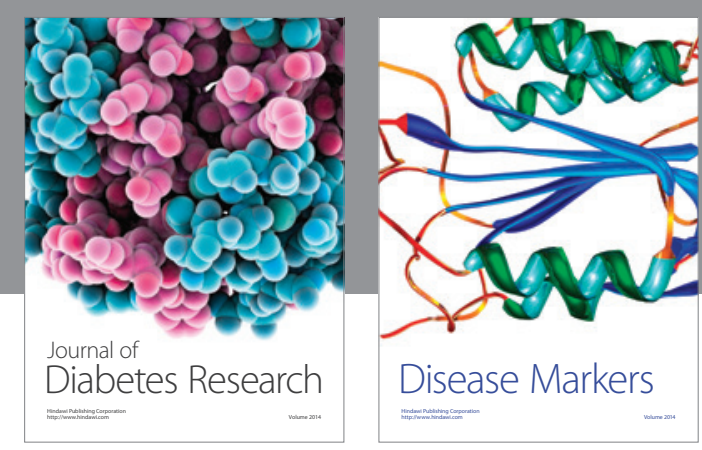

Disease Markers
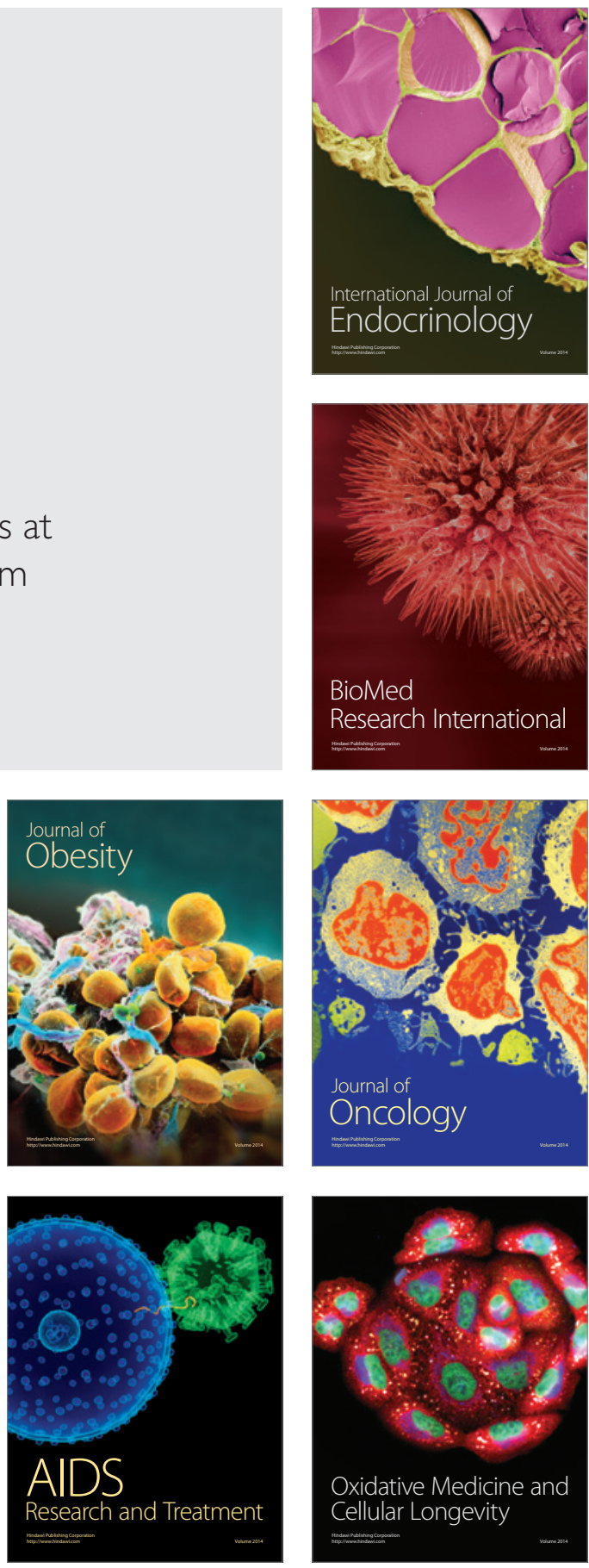